\title{
Multidisciplinary Cooperation in a Simultaneous Combined Liver and Kidney Transplantation Patient of Primary Hyperoxaluria 1
}

\author{
Qingqi Ren, ${ }^{1}$ Weiqiang Ju, ${ }^{2}$ Dongping Wang, ${ }^{2}$ Zhiyong Guo, ${ }^{2}$ Maogen Chen, ${ }^{2}$ Xiaoshun $\mathrm{He}^{2}$ \\ 'Department of hepatobiliary and pancreatic surgery, Peking University Shenzhen Hospital, Shenzhen China; ${ }^{2}$ Organ \\ Transplant Center, the First Affiliated Hospital, Sun Yat-sen University, Guangzhou, China.
}

\section{ABSTRACT}

Primary hyperoxaluria type 1 is an autosomal recessive hereditary glyoxylate metabolism disorder characterized by excessive production of oxalate, caused by the deficiency of liver specific peroxisomal enzyme: alanineglyoxylate aminotransferase. For patients with end-stage renal disease, combined liver and kidney transplantation was needed. This report describes one patient, with a diagnosis of end-stage renal disease and primary hyperoxaluria 1 confirmed by PCR and direct sequencing with genomic DNA, received the simultaneous combined liver and kidney transplantation after seven months' waiting. However, there were several complications observed post surgery, such as protracted bleeding, common bile duct anastomotic stenosis, biliary calculi and recurrence of urolithiasis. All these were well solved by relevant department, and finally a satisfactory outcome was achieved. Multidisciplinary cooperation plays an important role on the PH1 patient management, especially when multiple complications are encountered.

\section{Keywords: primary hyperoxaluria type 1; end-stage renal disease; liver transplantation; kidney} transplantation.

\section{INTRODUCTION}

Primary hyperoxaluria type $1(\mathrm{PH} 1)$ is a rare autosomal recessive hereditary disorder. For half of the patients, the diagnosis was made when the end-stage renal disease (ESRD) occurred and most of them were children. ${ }^{1}$ Some may even be diagnosed until urolithiasis recurrence after kidney transplantation. For patients with ESRD, combined liver and kidney transplantation (CLKT) was needed. ${ }^{2}$ Nowadays, improved knowledge of CLKT has been gotten. ${ }^{3,4}$ However, there is still a lack of reports of detailed complications and relative managements. We report a case with multiple complications and successful multidisciplinary cooperation on its managements.

\section{CASE REPORT}

A female girl, 11-year old, with a chief complaint of hematuria and repeated urolithiasis for 6 years, progressive urine decreasing and routine hemodialysis for 21 months, was admitted on December 2013. Serum creatinine maintained at the level of $459-838 \mu \mathrm{mol} / \mathrm{L}$ through hemodialysis every other day. The patient also suffered from secondary hypertension. X-ray radiograph showed calcification of bilateral kidney(Supplement Figure 1). The diagnosis of PH1 was confirmed by PCR and direct sequencing with genomic DNA. One known disease-causing frameshift duplication NM_000030.2 was detected, and also one novel nonsense mutation c. $242>$ A (p.Ser81).

After 7 months' waiting, a simultaneous CLKT, from a cadaveric donor, was carried out on July in 2014, and before the operation, a daily hemodialysis was accepted

Correspondence: Dr. Xiaoshun He, Organ Transplant Center, the First Affiliated Hospital, Sun Yat-sen University, Guangzhou, China. Email: xiaoshun_he@163.com, Phone: +086-13802510799. 
for consecutive 5 days to reduce the circulating oxalate maximally. Rabbit thymus lymphocyte globulin was used to induce immune tolerance at the dose of $1 \mathrm{mg}$ per kilogram body weight for 3 days. The operation lasted for 8 hours with a total blood loss of $150 \mathrm{ml}$. The patient was transferred to pediatric intensive care unit immediately after surgery to anesthesia resucitation and get back to organ transplant center after extubation. Mycophenolate mofetil, methylprednisolone and tacrolimus were used as prophylaxis against rejection. Pneumonia was treated by antibiotics. Antihypertensive agents were terminated 10 days later when blood pressure got back to normal range.

The serum total bilirubin increased gradually after surgery, while ultrasound test showed a intra hepatic cholangiectasis. Percutaneous transhepatic cholangical drainage (PTCD) was done under the guidance of ultrasound 12 days' post-surgery when the total bilirubin reached $339.6 \mu \mathrm{mol} / \mathrm{L}$ and it decreased to normal level one month later. X-ray showed anastomotic stenosis by infusion of contrast through PTCD tube (supplement figure 2). Then the PTCD tube was clamped from temporarily to persistently, neither jaundice nor fewer were observed and the tube was removed 36 days later.

Since the $5^{\text {th }}$ day, protracted bleeding was observed (figure 1) through the abdomen drainage tubes which resulted in a total 14 units of washed RBC and 1100 $\mathrm{ml}$ fresh frozen plasma transfused. It was especially obvious when the suture site, used to secure the PTCD tube, showed a persistent errhysis. A total of $9 \mathrm{KU}$ hemocoagulase was used intravenously and intramuscularly and $2 \mathrm{~g}$ tranexamic acid intravenously. Hemocoagulase also was also injected to the abdomen cavity directly through the drainage tubes and on the suture site.

A recurrence of urolithiasis occured both in the kidney and ureter (Figure 2) 60 days' post-surgery while the serum creatinine maintained at a level of $50-60 \mu \mathrm{mol} / \mathrm{L}$, accompanied by biliary calculi (Figure 3 ) due to its stricture. Biliary calculi were treated by therapeutic ERCP and stent implant due to repeated fever caused by the bile duct infection. Six biliary calculi, diameter ranging from 5 to 12 millimeters, were taken out. No recurrence was found during the next 8 months' follow up. The ureter calculi were removed using ureteroscope, and a double $\mathrm{J}$ tube was placed. An increase of serum creatinine $(133 \mu \mathrm{mol} / \mathrm{L})$ was observed due to the obstruction of the double $\mathrm{J}$ tube 5 months later, and the serum creatinine got back to normal after the replacement of double $\mathrm{J}$ tube and meanwhile the kidney stone was removed. The stone component of calcium oxalate monohydrate was confirmed through infrared spectrum detection.
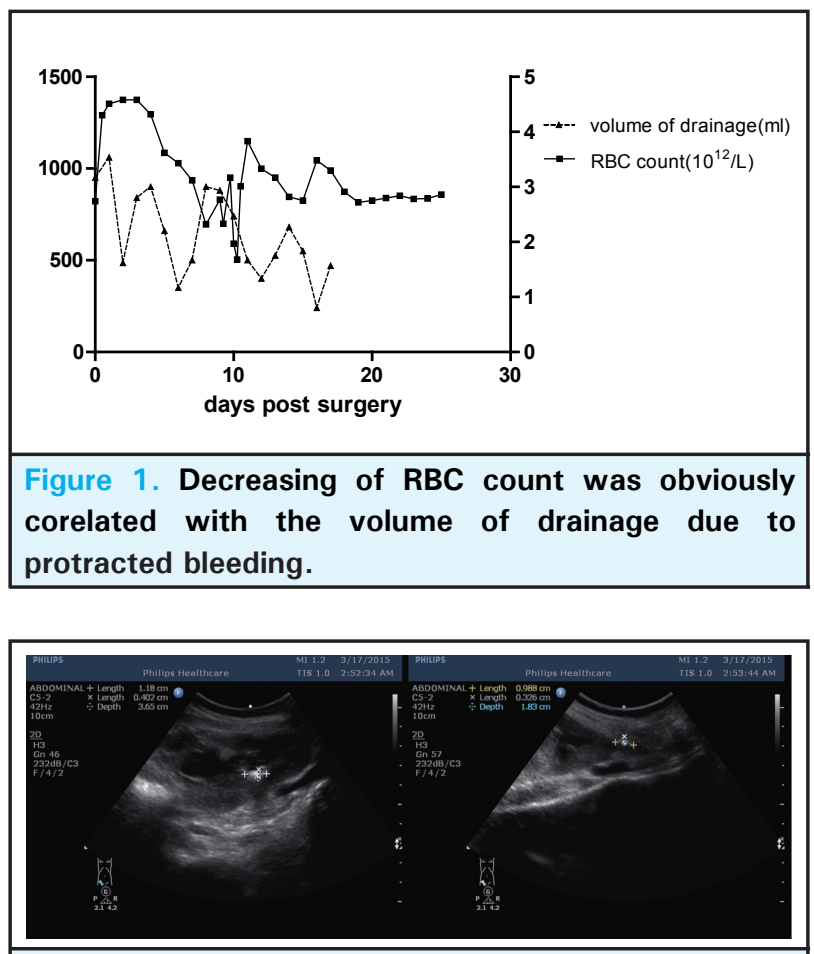

Figure 2. Recurrence of urolithiasis occured both in the kidney and ureter.

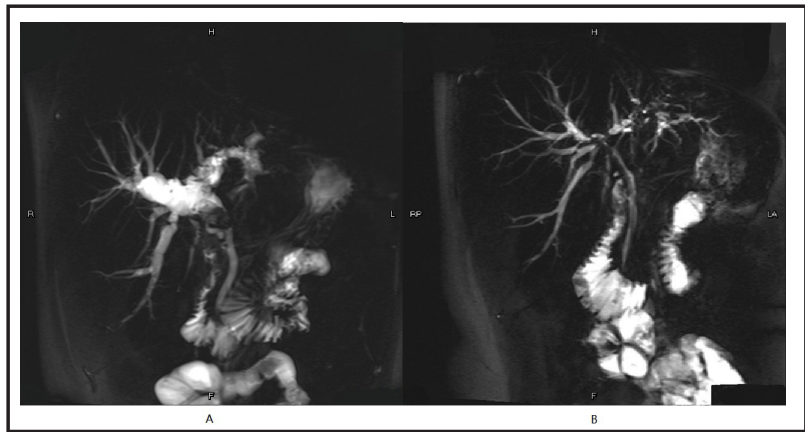

Figure 3. Biliary calculi caused by common bile duct anastomotic stenosis before(A) and after(B) therapeutic ERCP

\section{DISCUSSION}

This is the first patient receiving simultaneous liver and kidney transplantation in our center due to $\mathrm{PH} 1$. Both in the perioperative period and the follow-up, we encountered several complications, such as the protracted bleeding, common bile duct anastomotic stenosis, biliary calculi and recurrence of urolithiasis. Nowadays, there were about 10-30 pediatric CLKT every year, ${ }^{5}$ however, there was still lack of relative reports on treatment strategies in detail. Here, we described the complications and relative managements for future consideration.

As for the protracted bleeding, the role of oxalate calcium deposit in arterial media was ambiguous and 
another similarly case was reported by Watts RW et al, but without details. ${ }^{6}$ Hemocoagulase was used not only in the routine way, intravenously and intramuscularly, but also directly infused to the abdomen cavity through the drainage tube. An obvious reduction of errhysis was observed. Meanwhile, intensive examination of ultrasound and biochemical test were taken to monitor the blood flow of transplanted hepatic and renal vessels in case of thrombosis. To the biliary calculus caused by common bile duct anastomotic stenosis, we prescribed ursodesoxycholic acid to eliminate the calculus until repeated bile duct infection occurred, then the stones were removed through therapeutic ERCP and a plastic stent was placed across the site of stenosis. Ultrasound examination showed no evidence of calculus recurrence 8 months' post surgery, which also indicated that an early intervention to the stenosis may be beneficial.

Also, we made a retrospective analysis of plasma oxalate concentration by enzyme linked immunosorbent assay (ELISA) according to the manufacture's instructions, which showed a negative relationship with the urine volume, especially at the first month post surgery (supplement figure 3). The plasma oxalate concentration decreased to approximate normal level 4 months post surgery, which was identical to the previous report. ${ }^{7}$ Vitamin B6 and Potassium sodium hydrogen citrate were prescribed to prevent urolithiasis recurrence and a high volume of fluid intake to guarantee more than 2,000 $\mathrm{ml}$ urine (body surface area: $1.1162 \mathrm{~m}^{2}$ ) was required according to the 2009 KDIGO guideline. ${ }^{8}$ However, the patient still suffered from this complication, which may caused by the increasing of urine oxalate concentration due to the urine volume reduction post surgery. It was reported that the release of oxalate deposited in bone may last for up to 3 years in most patients, resulting in hyperoxaluria, especially in the first year following transplantation. ${ }^{7}$ Perioperative hemodialysis was also a concerning problem. Hemodialysis post operation was still in debating and it was omitted in this case due to the concern of oliguria as reported ${ }^{9}$ even when the urine volume reduced to $820 \mathrm{ml}$ a day and also due to the concurrent bleeding, which may be worsen by heparin used during hemodialysis and a lack of experience in hemodialysis on postoperative $\mathrm{PH} 1$ patients. An overall consideration of patients' specific condition and doctors' experience may facilitate decision making.

Now the patient has been back to school the first time after a 3-year's interruption with a normal liver function and creatinine clearance rate of $88.539 \mathrm{~mL} / \mathrm{min}$. Here, we highlight the multidisciplinary cooperation on the patient management. The department of organ transplant center, anesthesia, paediatric intensive care unit, hemodialysis center, interventional ultrasound, urological surgery and the department of interventional radiology department were all involved and integrated in the case and finally a satisfactory outcome was achieved.

\section{ACKNOWLEDGEMENT}

We acknowledge Jianwei Lin; Yujian Liang; Lu Yang; Xun Li.

\section{Conflict of Interest: None.}

Consent: JNMA Case Report Consent Form was signed by the patient and the original is attached with the patient chart.

\section{REFERENCES}

1. Cochat P, Koch Nogueira PC, Mahmoud MA, Jamieson NV, Scheinman JI, Rolland MO. Primary hyperoxaluria in infants: medical, ethical, and economic issues. The Journal of pediatrics. 1999;135(6):746-50. [Full Text | PubMed]

2. Cochat P, Hulton SA, Acquaviva C, Danpure CJ, Daudon M, De Marchi M, et al. Primary hyperoxaluria Type 1: indications for screening and guidance for diagnosis and treatment. Nephrol Dial Transplant. 2012;27(5):1729-36. [ [Full Text | PubMed]

3. Calinescu AM, Wildhaber BE, Poncet A, Toso C, McLin VA. Outcomes of combined liver-kidney transplantation in children: analysis of the scientific registry of transplant recipients. Am J Transplant. 2014;14(12):2861-8. [Full Text | PubMed]
4. Millan MT, Berquist WE, So SK, Sarwal MM, Wayman KI, Cox $\mathrm{KL}$, et al. One hundred percent patient and kidney allograft survival with simultaneous liver and kidney transplantation in infants with primary hyperoxaluria: A single-center experience. Transplantation. 2003;76(10):1458-63. [․ㅡll Text [ PubMed]

5. Jalanko H, Pakarinen M. Combined liver and kidney transplantation in children. Pediatr Nephrol. 2014;29(5):805-14. [Full Text | PubMed]

6. Watts RW, Danpure CJ, De Pauw L, Toussaint. Combined liver-kidney and isolated liver transplantations for primary hyperoxaluria type 1: the European experience. The European Study Group on Transplantation in Hyperoxaluria Type 1. Nephrol Dial Transplant. 1991;6(7):502-11. [ [Full Text [ PubMed] 
7. Bergstralh EJ, Monico CG, Lieske JC, Herges RM, Langman $\mathrm{CB}$, Hoppe B, et al. Transplantation outcomes in primary hyperoxaluria. Am J Transplant. 2010;10(11):2493-501. [피l Text $\mid \underline{\text { PubMed] }}$

8. Kasiske BL, Zeier MG, Chapman JR, Craig JC, Ekberg H, Garvey CA, et al. KDIGO clinical practice guideline for the care of kidney transplant recipients: a summary. Kidney Int. 2010;77(4):299-311. [ㅍll Text | PubMed]
9. Jouvet P, Priqueler L, Gagnadoux MF, Jan D, Beringer A, Lacaille F, et al. Crystalluria: a clinically useful investigation in children with primary hyperoxaluria post-transplantation. Kidney Int. 1998;53(5):1412-6. [Full Text | PubMed] 